\title{
Cat Swarm Optimization Algorithm For Antenna Array Synthesis
}

\author{
Nirmala Yerpula a, Dr. A A Ansari ${ }^{\text {b }}$ and Dr.M .Surendra Kumarc \\ a Research Scholar, Dept. of Electronics and Communication Engineering, \\ Sri Satya Sai University of Technology \& Medical Sciences, Sehore, Bhopal Indore Road, Madhya Pradesh, India \\ ${ }^{b}$ Research Guide, Dept. of Electronics and Communication Engineering, \\ Sri Satya Sai University of Technology \& Medical Sciences, Sehore, Bhopal Indore Road, Madhya Pradesh, India \\ ${ }^{c}$ Research Co-Guide, KLR College of Engineering and Technology, Palvoncha
}

Article History: Received: 11 January 2021; Accepted: 27 February 2021; Published online: 5 April 2021

\begin{abstract}
Cat swarm optimization (CSO) is a developmental technique enlivened by the animals in Mother Nature for taking care of optimization issue. Short of what multi decade after CSO is proposed, it has been improved and applied in various fields by numerous scientists as of late. CSO is created by noticing the practices of cats, and made out of two sub-models, i.e., following mode and looking for mode, which model upon the practices of cats. The prerequisite of high directivity signal with extremely quick pillar guiding is preposterous by a solitary antenna. This imperative is aid by staged array antenna which is a mix of various little antennas that can create shaft with high directivity with quick electronic pillar guiding. The radiation example of an antenna array relies firmly upon the weighting technique and the math of the array. The issues related with pillar design causing high obstruction in communication which confine them use by and by. To beat these im-pediments, optimization innovation called Cat Swarm Optimization are applied in mix with the old style array blend strategies for staged array combination. An optimization issue is determined whose arrangement yields an ideal array for stifling impedance because of high side lobe level and grinding lobe. Results are introduced for ideal arrays of shifting array calculation, with various number of antenna components, and for distinct beamwidths and scan angles.
\end{abstract}

Keywords: Cat swarm optimization, antenna array, tracing mode, seeking mode.

\section{Introduction}

In wireless communication, antenna is the cardinal construction in transmitter end just as at the beneficiary finish of the system. Antenna application got pervasive around world war-II. Just an antenna can make the communication system wireless, smart, portable, light weight, cost effective, more over made conceivable communication past the globe or across the seas or inside the body parts. Single antenna show low order acquire alongside different limits for application by and by. Idea of array antenna, a gathering of antennas work in harmony, first utilized in military application to improve communicated and got designs. Array antenna next, empowered with the system of electronic controlling so the array get or send signal towards a specific course without mechanical development, encourage quick communication. This array structure is named as staged array, by and large furnished with singular stage shifter with each array component, can be constrained by external computer. The radiation example of an array antenna can be constrained by altering the general current excitation, stage or dispersing between the components. Staged array enamors the high level sign handling innovation to communicate/get signal from a specific course while dismissing obstruction from undesirable heading. Another significant commonsense application of staged array antenna is sticking or purposeful impedance by nulling out radiation energy toward the ideal bearings.

Current cell communication requires area bar design radiation to cater more clients at the same time with great clearness of communication. This sort of shaft design achieves through staged array with cutting edge signal handling. Antenna design blend comprehensively categorized into three gatherings as indicated by pillar design, first gathering asking a bar design with nulls are in wanted ways, as Schelkunoff array, second gathering request shaft forming so the example show an ideal dispersion, similar to Woodward-Lawson array and the third gathering incorporates tight bar with low side projection level examples like binomial array, Tchebyscheff array and so forth Staged array creates shaft with high directivity, simultaneously offer ascent to high side flap level and expanding of bar during examining. Synthesis of enormous array includes a lot of complex sign handling work with the synchrony in supporting hardware to actualize the algorithms accordingly successful actual changes in array calculations get clouded. Practically speaking, array configuration incorporates loads of limitations and factors, definitely enormous arrays, the scientific and traditional strategies discovered wasteful because of high intricacy in optimization.

Adaptive array configuration is proposed by controlling impedance utilizing least square and least mean square algorithm. Direct programming based polynomial-time algorithm can be used for optimization of array amalgamation. A far reaching conversation is given about the engaged opening amplitude tightening and its impact on front lobes, back lobes, side lobesand gain of the array antenna is contrasted with deference with Taylor array tightening and uniform excitation. Side flap level of array antenna likewise relies upon components excitation stages. The perception in different instances of direct or planar array saw that logarithmic capacity of array size can decide the ideal side projection level which has more prominent proficiency than amplitude tightening. As the mathematical calculation is illogical for enormous array to discover singular stage for ideal side projection, an algorithm is proposed to figure stage dissemination of the array for ideal side flap level. Nonlinear amplitude optimization is a complicated cycle for array excitation, another idea showed that non- 
uniform component dividing either by customary diminishing or finite impulse responsefilter plan idea can create controlled side projection level for consistently excited array.

\subsection{Basic antenna models}

There are many variations of antennas. Few basic models are as follows.

- Dipole antenna: The dipole antenna is basically two wires pointed in inverse ways orchestrated either evenly or vertically, with one finish of each wire associated with the radio and the opposite end hanging free in space. Since this is the least complex viable antenna, it is additionally utilized as a source of perspective model for different antennas; acquire concerning a dipole is named as $\mathrm{dBd}$. By and large, the dipole is viewed as omnidirectional in the plane opposite to the pivot of the antenna, yet it has profound nulls in the ways of the hub. Varieties of the dipole incorporate the collapsed dipole, the half wave antenna, the ground plane antenna, the whip, and the J-pole.

- Random wire antenna:The arbitrary wire antenna is just an exceptionally long (in any event one quarter frequency wire with one end associated with the radio and the other in free space, organized in any capacity generally helpful for the space accessible. Collapsing will diminish adequacy and make hypothetical examination incredibly troublesome. Commonly, an arbitrary wire antenna will likewise require an antenna tuner, as it may have irregular impedance that fluctuates non-straightly with frequency.

- Isotropic radiator: The isotropic radiator is an absolutely hypothetical antenna that transmits similarly every which way. It is viewed as a point in space without any measurements and no mass. This antenna can't actually exist, yet is helpful as a hypothetical model for examination with any remaining antennas. Most antennas gains are estimated regarding an isotropic radiator, and are appraised in $\mathrm{dBi}$ (decibels as for an isotropic radiator).

- Parabolic antenna: The illustrative antenna comprises of a functioning component at the focal point of an explanatory reflector to mirror the waves into a plane wave. Like the horn, it is utilized for high addition, microwave applications, like satellite dishes.

- Yagi-Uda antenna:The Yagi-Uda antenna is a directional variety of the dipole with parasitic components added which are usefulness like add a reflector and focal points (chiefs) to shine a fiber light.

- Horn antenna: The horn antenna is utilized where high increase is required, the frequency is short (microwave) and space isn't an issue. Horns can be tight band or wide band, contingent upon their shape. A horn can be worked for any frequency, however horns for lower frequencies are normally unrealistic. Horns are additionally regularly utilized as reference antennas.

\subsection{Antenna Characteristics}

An antenna can be characterized as a momentary design between a guided medium and free space, moving data as emanated electromagnetic energy from transmitter to beneficiary. It is noticeable from the investigation of a little single antenna, creates consistently disseminated radiation fields, and furnishes more extensive bar with low directivity and gain. These antennas are appropriate for communicated administrations where wide inclusion required. At present age communication requests highlight point clamor less communication towards a favored heading. This can just accomplished by limited pillar exceptionally order antennas which turns out to be not really conceivable by a solitary little antenna to satisfy such necessities. A bigger size antenna can be a basic answer for expanding directivity however it isn't essentially feasible. One commonsense way to deal with plan antenna in an alternate manner that rather than one enormous antenna various little antennas are set together which is called as antenna array and commitment of every antenna are liable for a specific radiation design by the array. Since the initiation of electromagnetic radiation, the significance of antenna in wireless communication is expanding and, as of now, antenna application gets ubiquitous from biomedical to satellite communication. The essential square outline of wireless communication system is appeared in Figure 2.1.

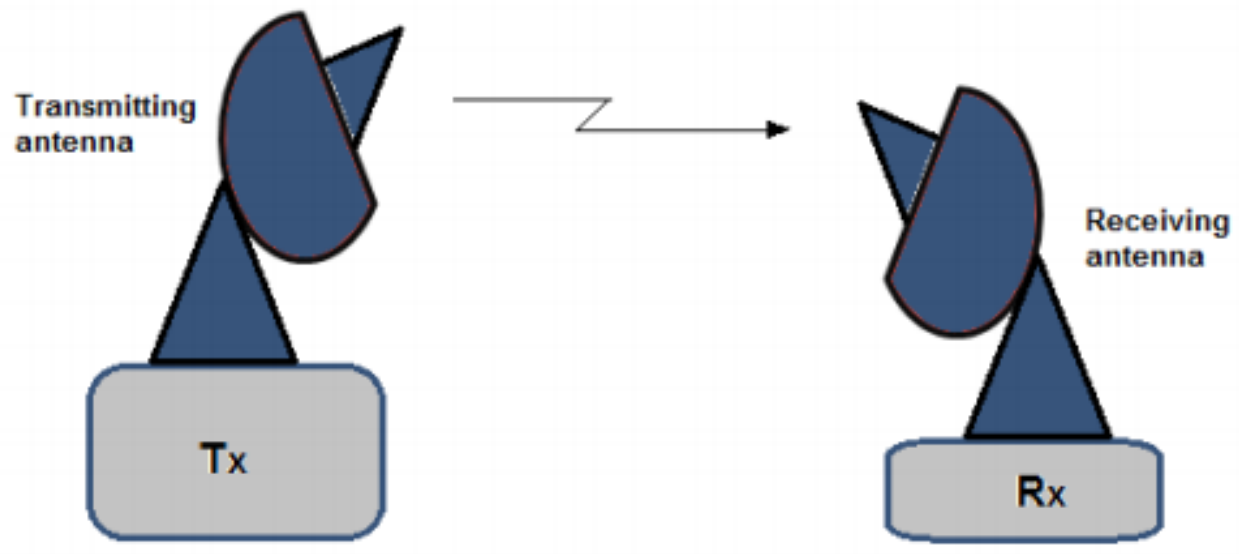

Figure 2.1. Block diagram of wireless communication 
The IEEE Standard (IEEE Std 145-183) characterizes antenna as, "a means for radiating and receiving radio waves." Thus antenna is a change gadget, changing over electrical energy from the transmitter into electromagnetic radiation which is going through the space, gotten by the accepting antenna at the recipient where it supplies identical electrical energy. A pictorial portrayal of antenna as communication connect between free space and transmission line is appeared in Figure 2.2.

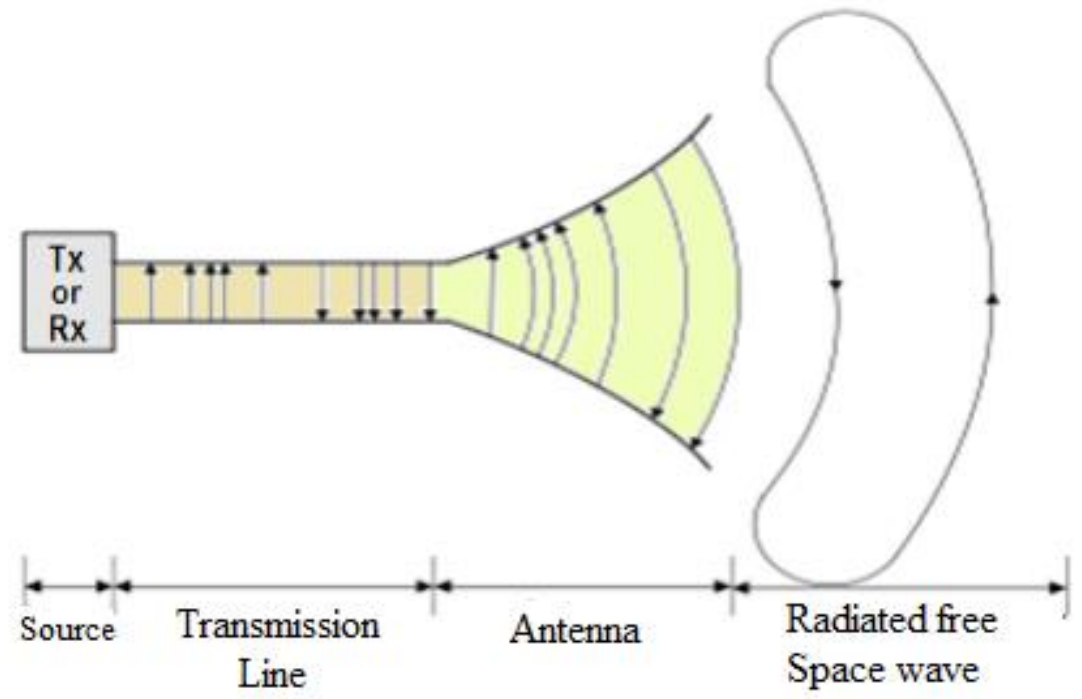

Figure 2.2. Antenna as a transitional device

The antenna radiation is following the rule that quickened charge or time changing current produces radiation field is opposite to the speeding up and can be communicated as,

$$
i L=Q v
$$

Where, $\mathrm{I}=$ time fluctuating current, $\mathrm{L}=$ length current component, $\mathrm{v}=$ quickening of charge and $\mathrm{Q}=$ charge. An array antenna is a mix of $N$ number of individual and comparable antennas (additionally called as components) which are isolated by a distance. Array antenna displays better and favorable quality analyzed than a solitary enormous antenna particularly with improved directivity and reasonable use.

\section{LITERATURE REVIEW}

Sudipta Banerjee et al (2020):In this paper the steerable isotropic roundabout array antenna is intended for lessening the side lobe level (SLL) utilizing transformative optimization method. The optimization strategies molecule swarm optimization and cat swarm optimization algorithm are utilized to lessen the SLLjust as to control the primary shaft explicit way. In this plan of steerable roundabout arrays the amplitude excitations are enhanced. Gotten results show that the greatest pinnacle of SLL of the resultant examples are according to prerequisite. This paper present a decent execution in the array factor reaction and stifled SLLfor various number of array components with various controlling point of the principle bar utilizing transformative optimization procedure.

Hrudananda Pradhan et al (2020): This paper depicts ideal antenna array structure for beamforming in brilliant antenna innovation. Various instances of array structures are investigated by the utilization of recently created optimization procedure called Whale Optimization Algorithm (WOA). The plan objective here has been considered to get the most positive radiation designs with sidelobe level (SLL) decrease by advancing amplitude excitations, bury component spacings and periods of every components of an array. The mathematical and reenactment results are contrasted and that got by most famous optimization method, Particle Swarm Optimization (PSO), Ant Lion Optimization (ALO) and Cat Swarm Optimization (CSO) by building regulations utilizing MATLAB. The outcomes show that WOA can display moderately preferable execution over different algorithms for the antenna array combination models and beamforming applications.

\section{PROPOSED METHODOLOGY}

Optimization is the interaction by which the ideal arrangement is chosen for a given issue among numerous elective arrangements. One central question of this cycle is the hugeness of the quest space for some genuine issues, in which it isn't practical for all answers for be checked in a sensible time. Nature-propelled algorithms are stochastic strategies, which are intended to handle these sorts of optimization issues. They as a rule incorporate some deterministic and haphazardness procedures together and afterward iteratively think about various arrangements until a palatable one is found. These algorithms can be categorized into direction based and populace based classes. In direction based sorts, like a mimicked strengthening algorithm, just a single specialist 
is looking in the hunt space to locate the ideal arrangement, though, in the populace based algorithms, otherwise called swarm Intelligence, for example, Cat swarm optimization (CSO), different specialists are looking and communicating with one another in a decentralized way to locate the ideal arrangement. Specialists for the most part move in two stages, in particular, investigation and misuse. In the first, they proceed onward a worldwide scale to discover promising territories, while in the subsequent one, they search locally to find better arrangements in those promising zones found up until now. Having a compromise between these two stages, in any algorithm, is extremely vital on the grounds that biasing towards one or the other investigation or misuse would debase the general exhibition and produce bothersome outcomes. Hence, more than many swarm insight algorithms have been proposed by analysts to accomplish this adjust and give better answers for the current optimization issues.

Optimization algorithms dependent on the Swarm Intelligence (SI) were created for recreating the shrewd conduct of creatures. In these displaying systems, a populace of organic entities like ants, honey bees, winged animals, and fish are communicating with each other and with their current circumstance through sharing data, bringing about utilization of their current circumstance and assets. One of the later SI-based optimization algorithms is the Cat Swarm Optimization (CSO) algorithm which depends on the conduct of cats. Created by Chu and Tsai (2007), the CSO algorithm and its assortments have been actualized for various optimization issues. CSO algorithm is made out of two modes, specifically following and looking for modes. Each cat addresses an answer set, which has its own position, a wellness esteem and a banner. The position is comprised of $\mathrm{M}$ measurements in the hunt space and each measurement has its own speed; the wellness esteem portrays how well the arrangement set (cat) is; lastly, the banner is to order the cats into one or the other chasing or following mode. Consequently, we should initially determine the number of cats ought to be occupied with the emphasis and run them through the algorithm. The best cat in every emphasis is saved into memory and the one at the last cycle will address the last arrangement.

\subsection{Natural Process of the Cat Swarm Optimization Algorithm}

Regardless of investing a large portion of their energy in resting, cats have high sharpness and interest in their environmental factors and moving items in their current circumstance. This conduct helps cats in discovering preys and chasing them down. Contrasted with the time dedicated to their resting, they invest too brief period on pursuing preys to ration their energy. Propelled by this chasing example, Chu and Tsai (2007) created CSO with two modes: "seeking mode" for when cats are resting and "tracing mode" for when they are chasing their prey. In CSO, a populace of cats are made and arbitrarily appropriated in the M-dimensional arrangement space, with each cat addressing an answer. This populace is separated into two subgroups. The cats in the main subgroup are resting and watching out for their environmental factors (i.e., seeking mode), while the cats in the subsequent subgroup begin moving around and pursuing their preys (i.etracing mode). The combination of these two modes encourages CSO to push toward the worldwide arrangement in the M-dimensional arrangement space. Since the cats invest too little energy in the following mode, the quantity of the cats in the following subgroup ought to be little. This number is characterized by utilizing the mixture ratio (MR) which has a little worth. Subsequent to arranging the cats into these two modes, new positions and wellness capacities will be accessible, from which the cat with the best arrangement will be saved in the memory. These means are rehashed until the halting models are fulfilled.

Following Chu and Tsai's, the computational strategies of CSO can be portrayed as follows:

Step 1:Create the underlying populace of cats and scatter them into the M-dimensional arrangement space (Xi,d) and velocity allot each cat a speed in scope of the velocity speed esteem (ti,d).

Step 2:According to the estimation of MR, appoint each cat a banner to sort them into the chasing or following mode measure.

Step 3:Evaluate the wellness estimation of each cat and save the cat with the best wellness work. The situation of the best cat (Xbest) addresses the best arrangement up until this point.

Step 4:Based on their banners, apply the cats into the chasing or following mode measure as depicted underneath.

Step 5:If the end measures are fulfilled, end the interaction. Otherwise repeat steps 2 through 5.

\begin{tabular}{|l|l|}
\hline General algorithm & Cat swarm optimization \\
\hline Decision variable & Cat's position in each dimension \\
\hline Solution & Cat's position \\
\hline Old solution & Old position of cat \\
\hline New solution & New position of cat \\
\hline Initial solution & Random positions of cats \\
\hline Best solution & Any cat with the best fitness \\
\hline
\end{tabular}




\begin{tabular}{|l|l|} 
Fitness function & Distance between cat and prey \\
\hline Selection & - \\
\hline Process of generating new solution & Seeking and tracing a prey \\
\hline
\end{tabular}

\subsubsection{Seeking Mode}

\section{Table 4.1 Characteristics of the CSO algorithm}

This mode emulates the resting conduct of cats, where four central boundaries assume significant parts seeking memory pool (SMP), seeking range of the selected dimension (SRD), counts of dimension to change (CDC), and self-position considering (SPC). These qualities are completely tuned and characterized by the client through an experimentation technique.

Looking for memory pool indicates the size of looking for memory for cats for example it characterizes number of competitor positions in which one of them will be picked by the cat to go to, for instance, in the event that seeking memory pool was set to 5 , for every single cat 5 new arbitrary positions will be created and one of them will be chosen to be the following situation of the cat. Instructions to randomize the new positions will rely upon the other two boundaries that are counts of dimension to change and self-position considering. CDC characterizes the number of measurements to be changed which is in the time period, $[0,1]$. For instance, if the pursuit space has 5 measurements and counts of dimension to change is set to 0.2 then for each cat four irregular measurements out of the five should be changed and the other one stays the equivalent. Self-position considering is the mutative proportion for the chose measurements for example it characterizes the measure of transformation and modifications for those measurements that were chosen by the CDC. At last, SPC is a Boolean worth, which determines whether the current situation of a cat will be chosen as a competitor position for the following emphasis, or not. Along these lines, for instance, on the off chance that the SPC banner is set to valid, for each cat, we need to produce (SMP-1) number of competitors rather than SMP numbers as the current position is considered as one of them. A portion of the fundamental elements identified with this mode are as per the following,

- Seeking memory pool (SMP): It tells how many number of copies of cat to be produced in seeking mode.

- Seeking range of the selected dimension (SRD): It tells how much range is varied for a selected dimension.

- Counts of dimension to change (CDC): It tells how many number of dimensions to be mutated.

Seeking mode steps are as follows:

Step 1:Make as numerous as SMP duplicates of the current situation of Catk.

Step 2:For each copy, randomly select as numerous as CDC measurements to be transformed. Additionally, haphazardly add or take away SRD values from the current qualities, which supplant the old situations as demonstrated in Equation 4.1.

$$
\mathrm{Xjd}_{\text {new }}=(1+\text { rand } * \mathrm{SRD}) * \mathrm{Xjd}_{\text {old }}
$$

Where $\mathrm{Xjd}_{\text {old }}$ is the current position; $\mathrm{Xjd}_{\text {newis }}$ is the following position; $\mathrm{j}$ signifies the quantity of a cat and $\mathrm{d}$ indicates the measurements; a rand is an arbitrary number in the time frame $[0,1]$

Step 3: Evaluate the fitness value (FS) for all the candidate positions.

Step 4:Based on probability select one of the up-and-comer focuses to be the following situation for the cat where up-and-comer focuses with higher FS have more opportunity to be chosen as demonstrated in Equation 4.2. In any case, in the event that all wellness esteems are equivalent, set all the choosing likelihood of every upand-comer highlight be 1 .

$$
\mathrm{Pi}=\frac{\left|\mathrm{FS}_{\mathrm{i}}-\mathrm{FS}_{\mathrm{b}}\right|}{\mathrm{FS}_{\max }-\mathrm{FS}_{\min }} \text {, where } 0<\mathrm{i}<\mathrm{j}
$$

If the objective is minimization then $\mathrm{FSb}=\mathrm{FSmax}$, otherwise $\mathrm{FSb}=\mathrm{FS} m i n$. 


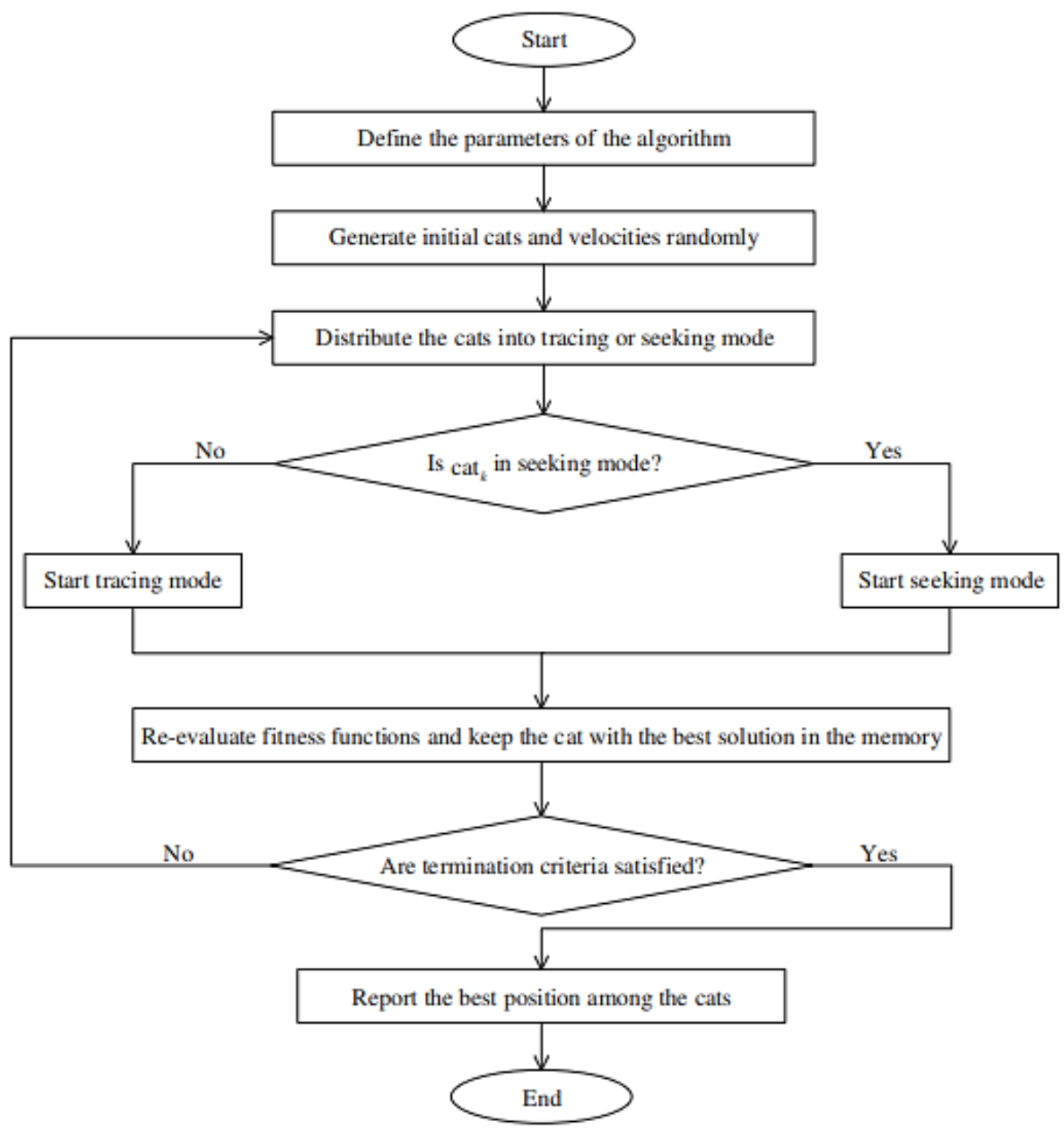

Figure 4.1 Flowchart of the CSO algorithm

\subsubsection{Tracing Mode}

This mode duplicates the following conduct of cats. For the main cycle, random velocity values are given to all elements of a cat's position. In any case, for later advances speed esteems should be refreshed. Moving cats in this mode are as per the following:

Step 1:Update speeds (Vk,d) for all measurements as indicated by Equation 4.3.

Step 2:If a speed an incentive out-ran the most extreme worth, at that point it is equivalent to the greatest velocity.

$$
\mathrm{V}_{\mathrm{k}, \mathrm{d}}=\mathrm{V}_{\mathrm{k}, \mathrm{d}}+\mathrm{r}_{1} \mathrm{c}_{1}\left(\mathrm{X}_{\mathrm{best}, \mathrm{d}}-\mathrm{X}_{\mathrm{k}, \mathrm{d}}\right)
$$

Where, vk,d = speed of cat $\mathrm{k}$ in measurement $\mathrm{d}$; Xbest,d = position of the cat with the best arrangement; Xk,d = position of the cat $\mathrm{k} ; \mathrm{c} 1=$ a consistent; and $\mathrm{r} 1=$ an arbitrary incentive in the scope of $[0,1]$.

Step 3: Update position of Catk according to Equation 4.4.

$$
X_{k, d, \text { new }}=X_{k, d, \text { old }}+v_{k, d}
$$

in which $\mathrm{Xk}$,d;new is the new position of cat $\mathrm{k}$ in dimension $\mathrm{d}$; and $\mathrm{Xk}$, d,old is the current position of cat $\mathrm{k}$ in dimension $\mathrm{d}$. 


\subsection{Applications of Cat Swarm Optimization}

Cat Swarm Optimization has been applied in science, innovation, business, space exploration and sociologies. Specialists and researchers have applied the ideas of CSO to different constant applications. There are numerous critical zones wherein the algorithm isn't been investigated, for instance; Medical Image Processing, Plant Monitoring, mechanical technology and so forth In this section, the applications of Cat Swarm Optimization from beginning to work date are dissected and nitty gritty data is introduced.

\subsubsection{Cat Swarm Optimization for Clustering}

Budi Santosa and Mirsa Kencana Ningrum created CSO bunching algorithm in 2009 and tried on four informational collections. They have adjusted the CSO recipe and contrasted and PSO and K-Means Clustering. The precision level of the proposed algorithm is reasoned by

- Clustering mistake for various number of cycles

- CPU time and grouping mistake of non-altered and changed cso bunching.

Every Method is iterated for 100 sudden spikes in demand for each dataset by the creators. The primary deduction of the technique is, however it is precise, it takes longer effort to do the calculation. Analysts can focus on instruments to build up a crossover technique for grouping genuine information from the genuine issue.

\subsubsection{Enhancement of ATC with STATCOM using Cat Swarm Optimization}

T.Nireekshana, Dr.G.KesavaRao and Dr.S.Siva Naga Raju created CSO as an apparatus to decide the location and controlling boundaries of STATCOM. Ideal position of Static Compensator, 6-Bus system, 14-Bus system, 26-Bus system, 30-Bus system, 6-Buses System with CSO, 30-Buses System with CSO were considered as contextual investigations to exhibit the improvement of ATC utilizing STATCOM.

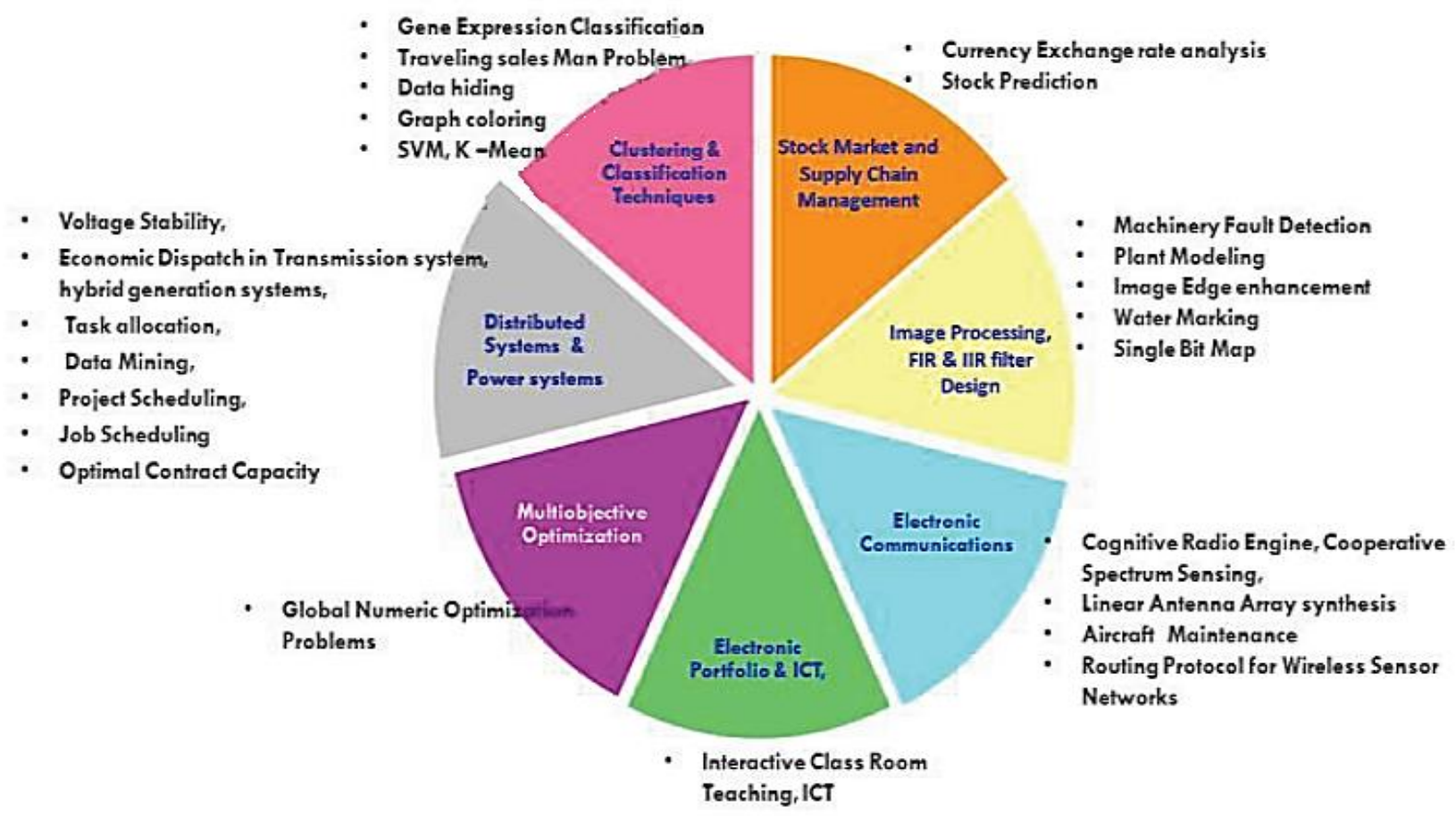

Figure 4.2 Applications of Cat Swarm Optimization

\subsubsection{Stock Market \& Supply Chain Management}

Suresh Chittineni picked PSO and CSO optimization algorithm to prepare the Neural Network for determining exactnesses of securities exchange. The creators guarantee that now and again PSO based NN outfoxed CSO based NN. The creators likewise presumed that the outcome isn't extremely exact on the grounds that stock rates rely upon conventional estimation factors as well as on different variables like policy driven issues, and any remaining issues identified with the organization which are hard to be quantized.

\subsubsection{Electronics \& Communication}

Pei-Wei Tsai created improved equal cat swarm optimization dependent on the Taguchi technique for taking care of airplane plan recuperation issue. To conquer the downsides of the customary CSO strategy and the PCSO technique, the creators received symmetrical array into the interaction of the equal following mode cycle to propose the improved equal cat swarm optimization (EPCSO) technique. The exploratory outcomes show that the proposed EPCSO strategy can give the ideal recuperated airplane plan for an extremely brief timeframe. The proposed EPCSO strategy gets a similar recuperation plan having a similar complete defer time, a similar deferred flight numbers and similar number of long postpone flights. 


\subsubsection{Image processing \& Filters}

Tirimula Rao Benala demonstrated that Hybrid channel by CSO upgrades the first picture proficiently when contrasted with smoothening channels and that got by the GA. The creators legitimize that in CSO the likelihood of falling into the neighborhood ideal is low a result of the blend of nearby and worldwide inquiry since the point of the algorithm is to improve the neighborhood search capacity of the GA without corrupting the worldwide pursuit capacity.

\section{RESULTS}

To accomplish most reduced pinnacle SLL and arrangement of nulls in wanted ways, CSO is applied to integrate the straight antenna array with the geography are acquired. Various kinds of direct array plans are thought of, some of which are like the plan models distributed in ongoing work and the relating component positions are advanced to achieve plan prerequisites while keep up uniform amplitude and stage excitations. The acquired outcomes are contrasted with the non-improved consistently divided array, alluded to in this after as customary array and furthermore to the PSO streamlined antenna array. It is seen from Figure 5.1, the CSO algorithm offers improvement in concealment of first side projection level by around $11.26 \mathrm{~dB}$ and $2.64 \mathrm{~dB}$ when contrasted with ordinary array math and PSO enhanced array separately.

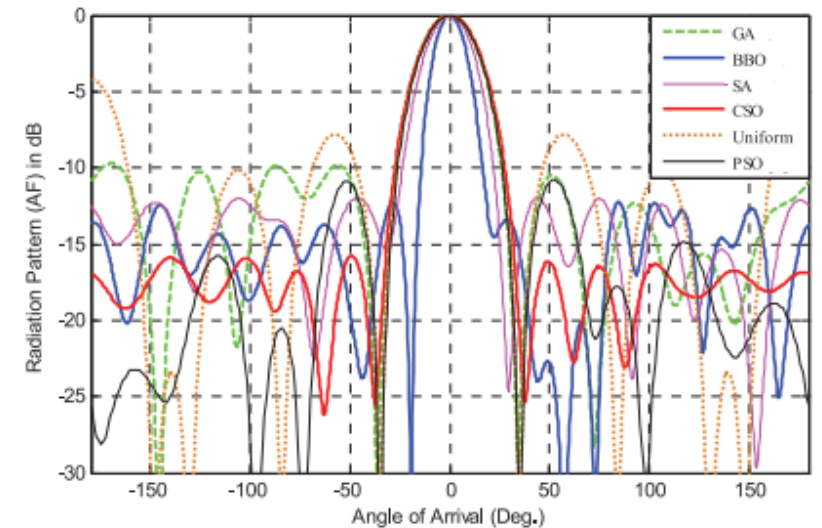

(a)

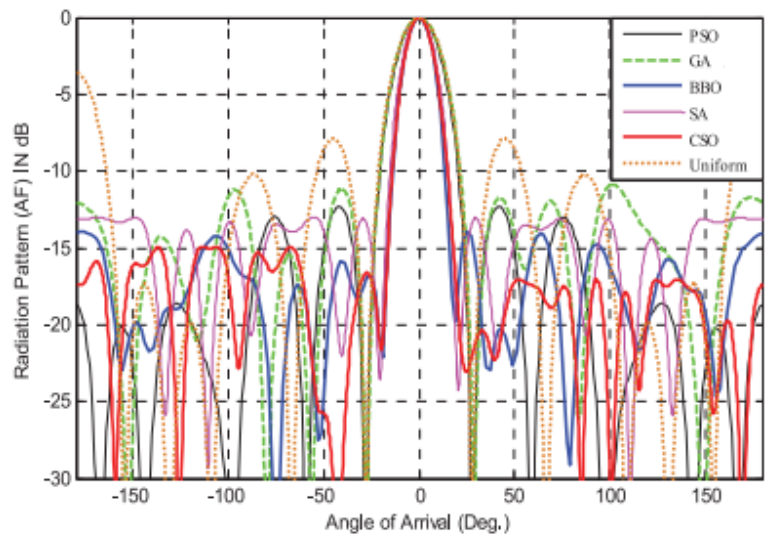

(b)

Figure 5.1. Comparative array patterns obtained by different algorithms for the (a) 8-element, (b) 10element

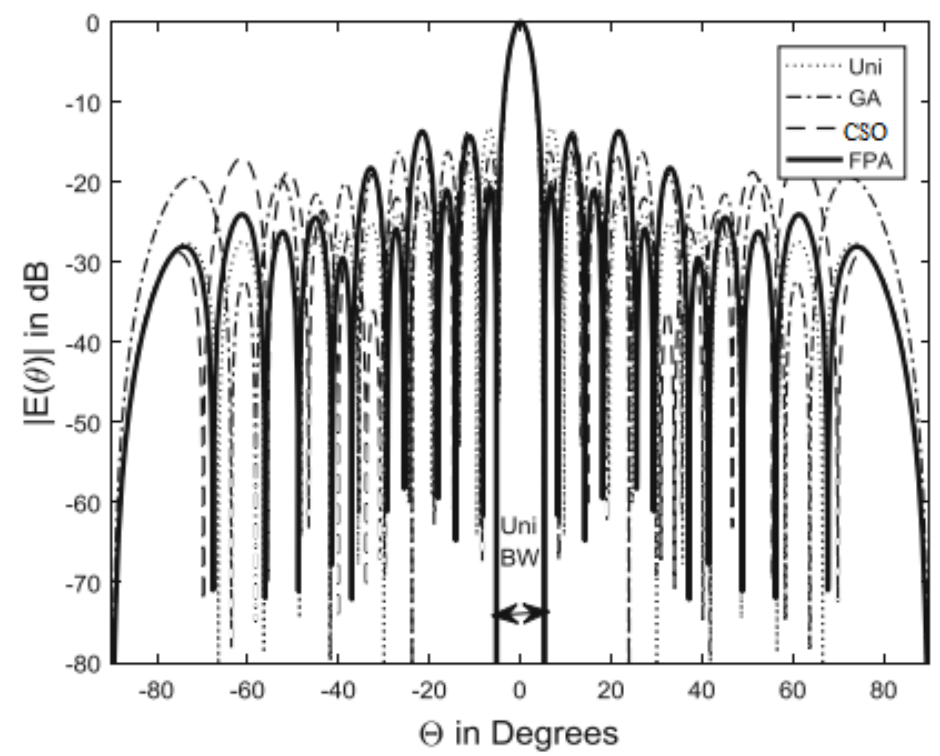

Figure 5.2 Comparison of SLL suppression for 24 element linear array sum patterns using GA, CSO and FPA with conventional uniform pattern (Amp-only technique)

The side-lobe levels of both total and contrast designs utilizing GA, CSO and FPA are combined. It is apparent that, with Amp-just strategy utilizing GA and CSO, the relating SLL in total examples is limited to -16.19 and $-16.31 \mathrm{~dB}$, individually, when contrasted and $-13.2 \mathrm{~dB}$ of regular uniform technique, though on application of 
FPA utilizing the Amp-just method, the SLL is brought down to $-16.86 \mathrm{~dB}$ keeping up a similar BW. It can likewise be construed that on account of FPA, the Amp-Sp strategy further decreased the SLL to $-28.86 \mathrm{~dB}$ which is obviously superior to the Amp as it were. Comparable pattern can be seen even with GA and CSO too.

\section{CONCLUSION}

In the investigation, in light of the advantages of CSO an Adaptive Cat Swarm Optimization (ACSO) algorithm is gotten. Through the cat swarm conduct in the following mode, there is a versatile acclimation to its boundaries. The viability of it has been tried through different straight array component capacities. This exploratory outcome indicates that CSO has brilliant execution than other existing heuristics during the time spent investigation and abuse. The bound together methodology of array combination has been proposed for both entirety and distinction designs in direct arrays. It is seen that the Amp-just procedure yielded helpless union rate when contrasted with the Amp-Sp-based strategy. CSO showed its capacity in handling unique and complex issues in different regions. Notwithstanding, much the same as some other meta-heuristic algorithm; the CSO algorithm has qualities and shortcomings. The Tracing mode looks like the worldwide inquiry measure while the looking for mode takes after the neighborhood search measure. This algorithm appreciates a huge property for which these two modes are isolated and autonomous. Notwithstanding, the algorithm has a high possibility of falling into nearby optima, known as untimely combination, which can be considered as the fundamental downside of the algorithm.

\section{REFERENCES}

1. Banerjee, S., \& Mandal, D. (2020). Array Pattern Optimization for Steerable Circular Isotropic Antenna Array Using Cat Swarm Optimization Algorithm. Wireless Personal Communications, 99(3), 11691194. doi:10.1007/s11277-017-5171-6

2. Pradhan, H., Mangaraj, B. B., \& Kumar Behera, S. (2020). Antenna Array Optimization for Smart Antenna Technology using Whale Optimization Algorithm. 2019 IEEE Indian Conference on Antennas and Propogation (InCAP). doi:10.1109/incap47789.2020.9134644

3. Budi Santosa and Mirsa Kencana Ningrum, "CSO Clustering" (2009). IEEE international conference of soft computing and patterns Recognition, pp 54-59

4. T.Nireekshana, Dr.G.KesavaRao and Dr.S.Siva Naga Raju,'Enhancement of ATC with STATCOM using Cat Swarm Optimization." ELSVIER Transactions on Proceedings of International Conference on Control, Communication and Power Engineering. Vol. 2, pp:422-428, 2013.”.

5. Suresh Chittineni, Vabbilisetty Mounica, Kaligotla Abhilash, Suresh Chandra, "A comparative study of CSO and PSO trained trained ANN for stock market prediction". International Conference on Computational Science, Engineering and Information Technology, Springer, Berlin, Heidelberg, pp. 186195,2020

6. Tsai, Pei-Wei, Jeng-Shyang Pan, Shyi-Ming Chen, and Bin-Yih Liao. "Enhanced parallel cat swarm optimization based on the Taguchi method." Expert Systems with Applications 39, no. 7 pp: 6309-6319, 2019

7. Tirimula Rao Benala, S. C. Satapathy, S. G. S. Surya Vamsi, Ravi, "CSO for Optimizing Hybridized Smoothening Filter In Image Edge Enhancement", International conference on Systemics, Cybernetics and Informatics, pp:247-252, 2020.

8. Hadi I, Sabah M. Improvement cat swarm optimization for efficient motion estimation. Int. J. Hybrid Inf. Technol. 2019;8(1):279-94.

9. Mallikalava, V., Yuvaraj, S., Vengatesan, K., Kumar, A., Punjabi, S., \& Samee, S. (2020). Theft Vehicle Detection Using Image Processing integrated Digital Signature Based ECU. In 2020 Third International Conference on Smart Systems and Inventive Technology (ICSSIT) (pp. 913-918).

10. El-Ela AA, El-Sehiemy RA, Kinawy AM, Ali ES. Optimal placement and sizing of distributed generation units using different cat swarm optimization algorithms. In2016 Eighteenth International Middle East Power Systems Conference (MEPCON) 2019 Dec 27 (pp. 975-981). IEEE.

11. Crawford B, Soto R, Berrios N, Olguin E. Solving the set covering problem using the binary cat swarm optimization metaheuristic. World Academy of Science, Engineering and Technology, International Journal of Mathematical, Computational, Physical, Electrical and Computer Engineering. 2020 Feb 5;10(3):104-8.

12. Vengatesan, K., Kumar, A., Naik, R., \& Verma, D. (2018). Anomaly based novel intrusion detection system for network traffic reduction. In 2018 2nd International Conference on I-SMAC (IoT in Social, Mobile, Analytics and Cloud)(I-SMAC) I-SMAC (IoT in Social, Mobile, Analytics and Cloud)(ISMAC), 2018 2nd International Conference on (pp. 688-690). 